\title{
In situ analysis of lithium-ion secondary battery using low-energy X-ray microscopy
}

\author{
Suguru UEMURA*, Takashi SASABE*, Yuichiro TABUCHI**, Junko KURIHARA***, Ting Chu JAO* \\ and Shuichiro HIRAI* \\ * Department of Mechanical Engineering, Tokyo Institute of Technology \\ 2-12-1 Ookayama, Meguro-Ku, Tokyo 152-8550, Japan \\ E-mail: uemura@mech.titech.ac.jp \\ ** Nissan Motor CO., LTD. \\ 1, Natsushima-cho, Yokosuka-shi, Kanagawa 237-8523, Japan \\ *** Automotive Energy Supply Corporation \\ 2-10-1 Hironodai, Zama-shi, Kanagawa 252-0012, Japan
}

Received: 16 October 2018; Revised: 25 January 2019; Accepted: 3 March 2019

\begin{abstract}
Lithium-ion secondary batteries have become key devices for energy storage for automotive and renewable energy applications. To further improve battery performance, clarification of the ion transport phenomena within the batteries is necessary. However, experimental investigation has not yet been performed. In this study, in situ visualization of lithium-ion batteries with a hard carbon or graphite negative electrode was achieved using low-energy X-ray microscopy. Visualization experiments were conducted on two different negative electrode materials. The concentration distributions of lithium ions $\left(\mathrm{Li}^{+}\right)$and hexafluorophosphate ions $\left(\mathrm{PF}_{6}^{-}\right)$inside the hard carbon negative electrode were investigated. Additionally, stage transformation caused by lithium intercalation was observed in the graphite negative electrode. These results suggest that transport phenomena in the electrodes of operating lithium-ion batteries can be investigated using low-energy X-ray microscopy.
\end{abstract}

Keywords: Lithium-ion secondary battery, Low-energy X-ray, Ion concentration, Electrode, Hard carbon, Graphite

\section{Introduction}

Recently, the demand for electric vehicles (EVs) using lithium-ion secondary batteries (LIBs) has rapidly increased. However, improvement of LIB performance is still required. To further incentivize the use of LIBs as a power source for EVs, it is important to improve the battery capacity and durability, realize fast charge, and maintain stable low-temperature operation.

Figure 1 presents a schematic image of a LIB. The fundamental structure of a LIB consists of a positive electrode, a separator, and a negative electrode. The electrode materials consist of microstructured or nanostructured porous media, in which the pores are filled by an electrolyte. The electrolyte is generally a solution of $\mathrm{LiPF}_{6}$. Most $\mathrm{LiPF}_{6}$ exists as $\mathrm{Li}^{+}$and $\mathrm{PF}_{6}{ }^{-}$ions in the electrolyte, and these ions migrate through the electrolyte during charging and discharging. During charging, lithium deintercalates from the active material of the positive electrode and intercalates into the active material of the negative electrode. As a result, an ion concentration gradient is induced in the electrolyte, with a high concentration on the positive-electrode side and a low concentration on the negative-electrode side.

The gradient of the ion concentration indicates the magnitude of the ion transport resistance in the electrolyte and is important for analyzing the essential charge and discharge characteristics of the battery. Furthermore, as the electrolyte concentration increases, the viscosity increases and the ion diffusion coefficient decreases, drastically increasing the ion transport resistance. Thus, determination of the ion concentration distribution is important for performance improvement of LIBs. 
(a) Open circuit

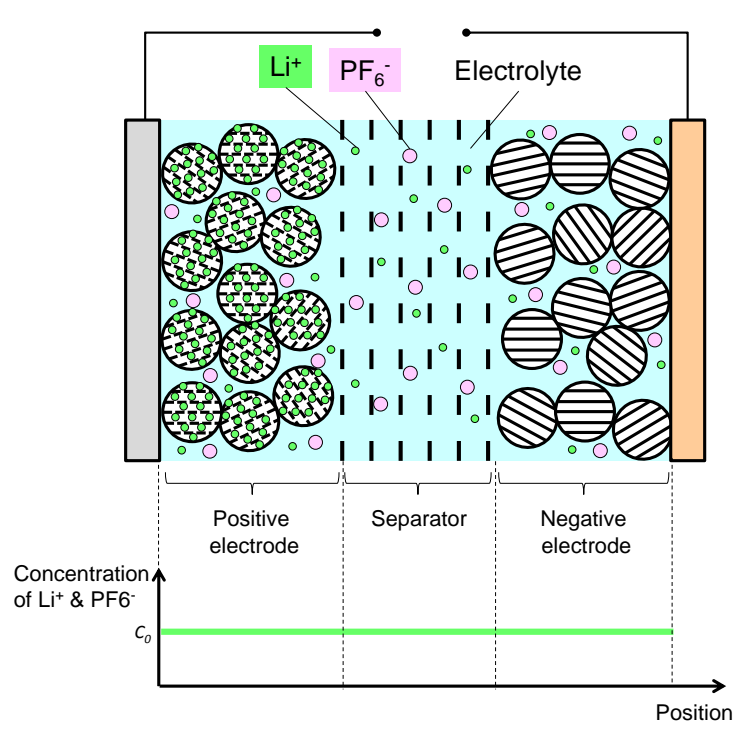

(b) Charging

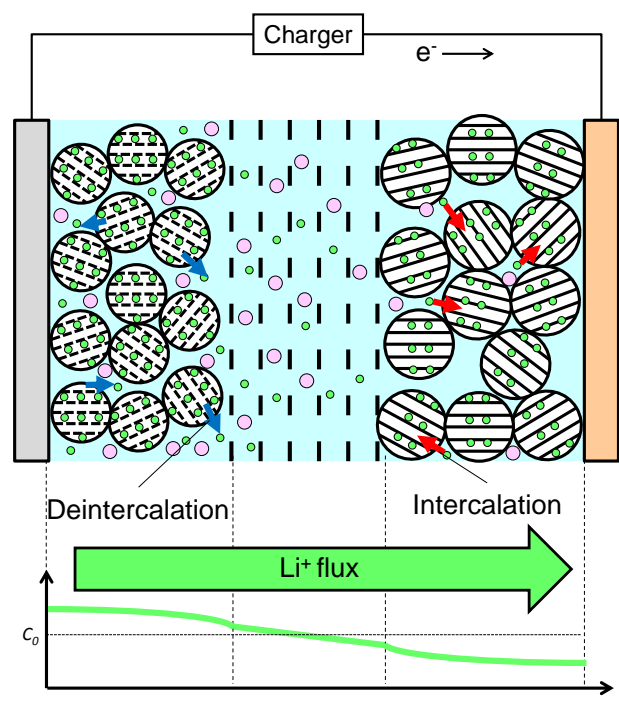

Fig. 1 Schematic image of LIB.

(a) Experimental setup

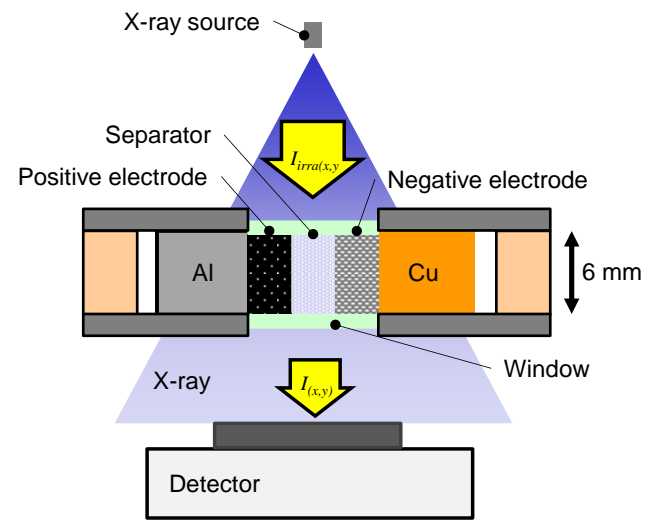

(b) LIB cell for visualization

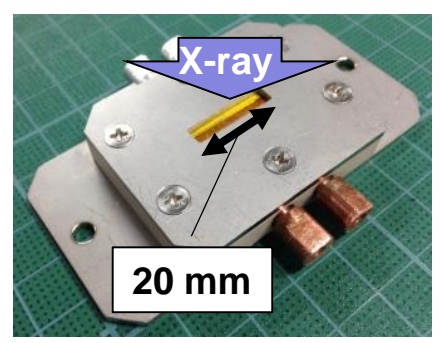

(c) X-ray transmission image

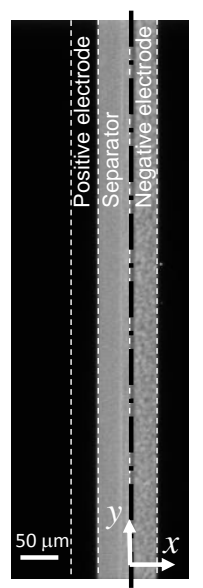

Fig. 2 Experimental apparatus.

Recently, numerous in situ investigations have been conducted to improve battery performance. X-ray and neutron techniques have been used for in situ and nondestructive observation of LIBs, respectively (Ebner et al., 2013, Harks et al., 2015, Bond et al., 2016). The three-dimensional nanoscale structures of the battery components were clearly visualized using computed tomography (CT) (Tariq et al., 2014). The microscale particle shape and its distribution in the active material and binder of the electrode were investigated. A low-energy X-ray microscopy technique to visualize the lithiation process in a lithium-ion half-cell has also been developed (Tsushima et al., 2015).

Considerable efforts are currently being directed toward nanoscale visualization. However, the fundamental ion transport phenomena occurring during LIB operation, which determine the battery performance, are not being investigated. In this study, in situ visualization of an operating (charging/discharging) LIB was achieved using a low-energy X-ray microscopy technique. The CT method requires a long imaging time; however, X-ray transmission imaging allows observation inside the battery in the operating state with high temporal resolution. Changes in the distribution of the ion concentration within the electrolyte inside the operating LIB as well as the shape deformation of the electrode structure due to intercalation were observed. 


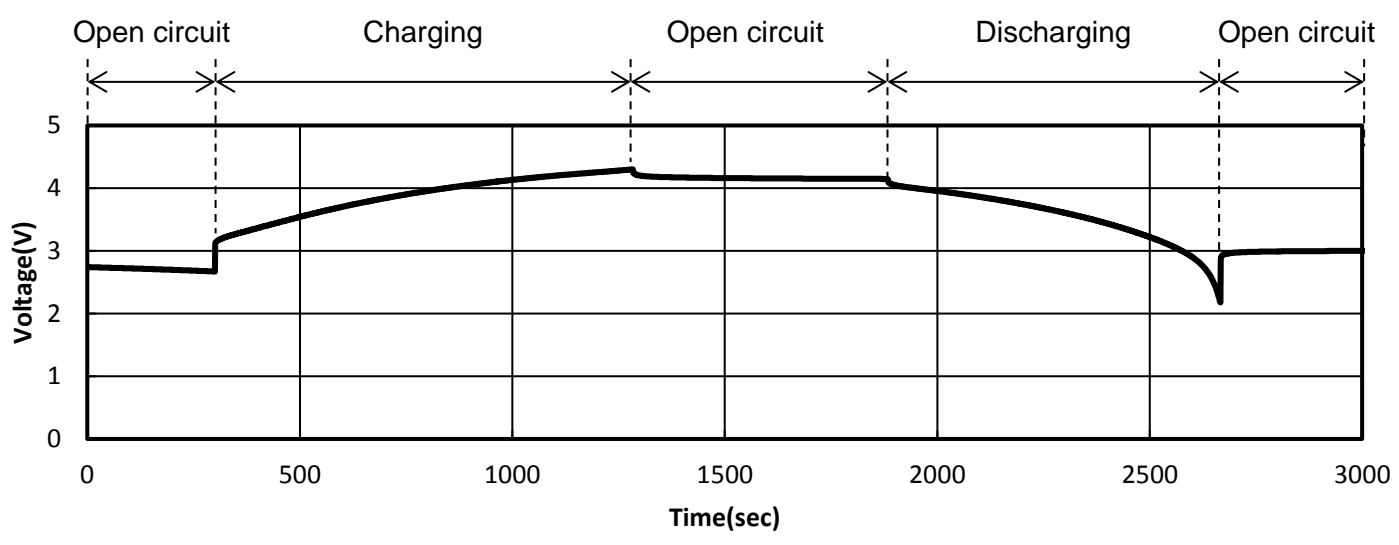

Fig. 3 Charge/discharge characteristics of LIB for visualization using hard carbon electrode.

\section{Experimental Methods}

\subsection{X-ray imaging system and visualization cell}

Figure 2 presents a schematic image of the experimental apparatus. A low-energy X-ray microscopy system (TUX-3110, Mars Tohken Solution Co. Ltd., Japan) was used in this study. To observe the transport phenomena in a thin microscale layer of the electrode and separator, high magnification (resolution of $1 \mu \mathrm{m} / \mathrm{pixel}$ ) was achieved by irradiating each LIB with a cone-shaped X-ray beam. To obtain a clear visualization, 12 individual images with an exposure time of $20 \mathrm{~s}$ each were integrated.

The cells for visualization, $\mathrm{LiMn}_{2} \mathrm{O}_{4}$ positive electrodes, negative electrodes (detailed below), and polypropylene microporous membrane separators with dimensions of $6 \times 20 \mathrm{~mm}$ were punched out using a Thomson die cutting machine. The components were sandwiched between aluminum and copper current collectors in a plastic frame. After injection of the electrolyte [1 $\mathrm{M} \mathrm{LiPF}_{6} / \mathrm{EC}: \mathrm{DEC}$ (3:7)], each cell was sealed with stainless-steel plate coverings that contained X-ray transparent windows. The entire assembly process was performed in an argon-filled glove box.

Two different active materials, hard carbon and graphite, were used as negative electrodes. The hard carbon negative electrode was prepared by mixing hard carbon (particle size: $10 \mu \mathrm{m}$ ), a conductive assistant, and polyvinylidene fluoride as a binder. The graphite electrode was prepared by mixing graphite (particle size: $20 \mu \mathrm{m}$ ), a conductive assistant, and the polyvinylidene fluoride binder. Figure 2(c) presents the X-ray transmission image obtained for the LIB with the hard carbon electrode.

Figure 3 shows an example of the charging and discharging characteristics of the LIB used for visualization with the hard carbon electrode. The charging/discharging rate was $3 \mathrm{C}$ (where $1 \mathrm{C}$ is the current rate that can fully charge or discharge the capacity of the battery in $1 \mathrm{~h}$ ). The voltage increased and decreased smoothly during the charging and discharging processes. The open-circuit voltage (OCV) also appeared to be stable after charge and discharge. These results indicate that the LIB used for visualization in this study operated normally.

\subsection{X-ray imaging of the LIB}

The luminance value at each pixel in the X-ray transmission image corresponds to the quantity of X-rays that reach each detector pixel from the X-ray source. When X-rays penetrate a substance, their absorption depends on the X-ray absorption coefficient and thickness of the substance, and the X-ray intensity attenuates exponentially. The relationship between the irradiated and transmitted X-ray intensities is described by the Beer-Lambert law as

$$
I_{(x, y)}=I_{i r(x, y)} \exp (-\mu L)
$$

where $I_{i r(x, y)}$ is the irradiated X-ray intensity, $I_{(x, y)}$ is the transmitted X-ray intensity, $\mu$ is the linear absorption coefficient of the substance, and $L$ is the thickness of the substance. The X-ray intensity at each pixel location $(x, y)$ in the transmission image can be expressed by Eq. (1). 


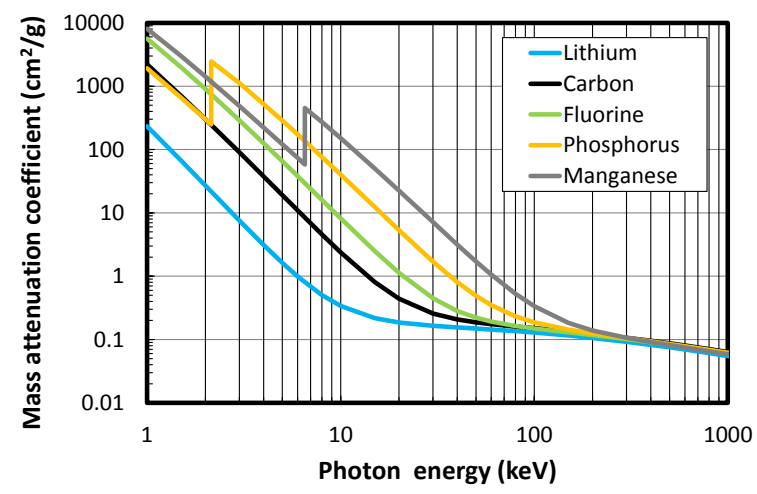

Fig. 4 Mass attenuation coefficients of the primary substances in LIB.

Equation (1) assumes that the X-rays pass through a single substance. However, the LIB electrode contains multiple substances: the active material and electrolyte. Thus, the relationship between the X-ray intensity at each pixel in the captured X-ray transmission image of the electrode is given by Eq. (2).

$$
I_{(x, y)}=I_{i r(x, y)} \exp \left\{-\mu_{A} L(1-\phi)-\mu_{E} L \phi\right\}
$$

In Eq. (2), the subscripts $A$ and $E$ represent the active material and electrolyte, respectively. The variable $\phi$ represents the porosity of the electrode.

Figure 4 presents the $\mu$ values for the primary materials in LIBs. The vertical axis, the mass attenuation coefficient, is a physical property that indicates how many X-rays can pass through a substance and is expressed by dividing the linear absorption coefficient by the density of the substance. In the conventional X-ray range (where the photon energy is greater than $100 \mathrm{keV})$, all the $\mu$ values are small; therefore, it is difficult to detect the change in $I_{(x, y)}$ caused by either the variation of the electrolyte concentration or the deformation of the electrode. The use of low-energy X-rays (1-10 $\mathrm{keV}$ ) instead is an effective method to improve the detection ability.

The $\mu$ value of lithium is small even in the low-energy X-ray range; therefore, it is difficult to detect any variation in the lithium ion $\left(\mathrm{Li}^{+}\right)$concentration in the electrolyte. However, because of the principle of electroneutrality, the hexafluorophosphate $\left(\mathrm{PF}_{6}{ }^{-}\right)$distribution is the same as the $\mathrm{Li}^{+}$distribution, and phosphorus and fluorine absorb $\mathrm{X}$-rays well. Therefore, the concentration distribution caused by the charge and discharge of $\mathrm{PF}_{6}^{-}$in the electrolyte can be determined from the change in the X-ray transmission intensity $I_{(x, y)}$, and the $\mathrm{Li}^{+}$concentration distribution can subsequently be determined.

The small X-ray absorption coefficient of lithium ions causes an additional problem. During charging, lithium is accumulated in the active material by intercalation; however, the presence of lithium is difficult to detect. Nevertheless, because the carbon of the active material expands during the intercalation, the accumulation of lithium can be detected from the deformation of the electrode structure. Observations of the positive electrode presented additional difficulties because the manganese contained in the active material significantly absorbs X-rays.

As previously mentioned, changes in the ion concentration in the electrolyte, intercalation of lithium into the active material, and changes in the void structure are the main phenomena that occur in the electrode during charging and discharging. Consequently, the X-ray transmission intensity can be expressed as

$$
I_{(x, y)}^{\prime}=I_{i r(x, y)} \exp \left\{-\mu_{A}^{\prime} L\left(1-\phi^{\prime}\right)-\mu_{E}^{\prime} L \phi^{\prime}\right\}
$$

where the prime indicator represents the variable after charge/discharge. By dividing Eq. (3) by Eq. (2), the change in the transmitted X-ray intensity is obtained, as expressed by Eq. (4). 
(a) 3C charge/discharge

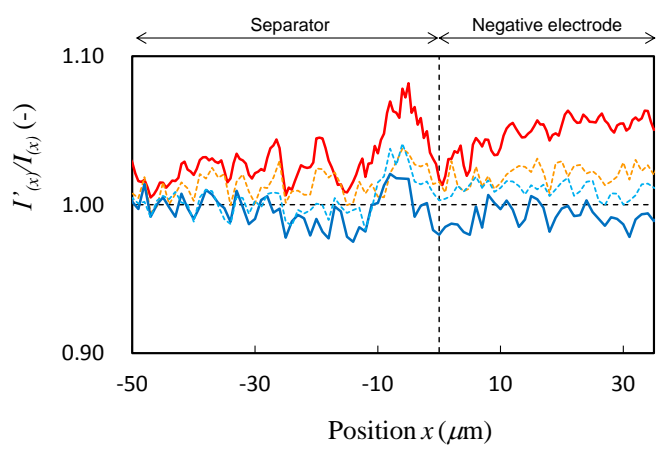

(b) 10C charge/discharge

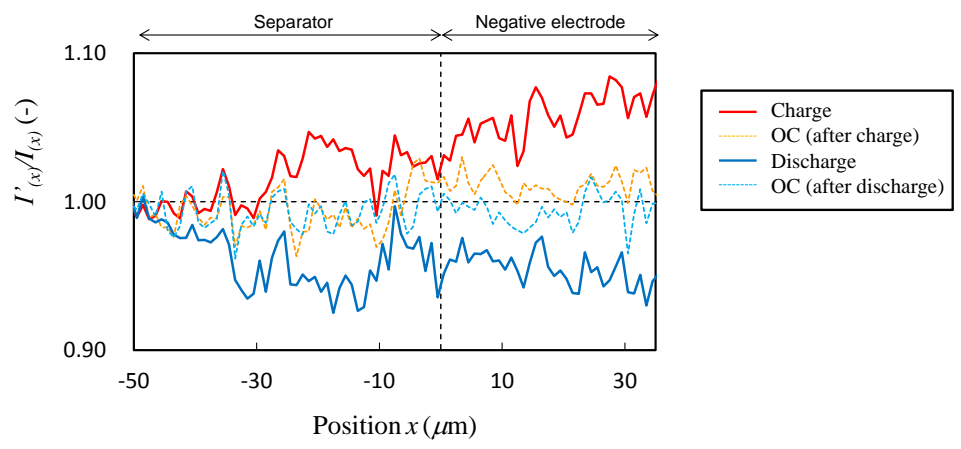

Fig. $5 I_{(x)}^{\prime} / I_{(x)}$ profiles for hard carbon negative electrode and separator at charge/discharge rates of (a) $3 \mathrm{C}$ and (b) $10 \mathrm{C}$.

$$
\frac{I_{(x, y)}^{\prime}}{I_{(x, y)}}=\frac{I_{i r(x, y)} \exp \left\{-\mu_{A}^{\prime} L\left(1-\phi^{\prime}\right)-\mu_{E}^{\prime} L \phi^{\prime}\right\}}{I_{i r(x, y)} \exp \left\{-\mu_{A} L(1-\phi)-\mu_{E} L \phi\right\}}=\exp \left[-\left\{\mu_{A}^{\prime} L\left(1-\phi^{\prime}\right)-\mu_{A} L(1-\phi)\right\}-\left(\mu_{E}^{\prime} L \phi^{\prime}-\mu_{E} L \phi\right)\right]
$$

The ratio $I_{(x, y)}^{\prime} / I_{(x, y)}$ indicates the state change induced in the electrode. When the electrolyte concentration and porosity change simultaneously during charging and discharging, the resulting change in $I_{(x, y)}^{\prime} / I_{(x, y)}$ cannot be used to distinguish between these phenomena. Therefore, hard carbon was used as the negative electrode, and appropriate experimental conditions were applied. Because hard carbon deforms less upon charging and discharging $\left(\mu_{\mathrm{A}}{ }^{\prime}=\mu_{\mathrm{A}}\right)$ and the porosity change can be neglected $\left(\phi^{\prime}=\phi\right), I_{(x, y)}^{\prime} / I_{(x, y)}$ can be written as

$$
\frac{I_{(x, y)}^{\prime}}{I_{(x, y)}}=\exp \left\{-\left(\mu_{E}^{\prime}-\mu_{E}\right) L \phi\right\}
$$

The difference in the X-ray absorption coefficient $\left(\mu_{E}^{\prime}-\mu_{E}\right)$ qualitatively indicates the ion concentration difference in the electrolyte, and $L$ and $\phi$ are known values. Thus, the qualitative ion concentration distribution in the electrode can be determined by measuring the ratio $I_{(x, y)}^{\prime} / I_{(x, y)}$. Because no deformation occurs in the separator, Eq. (5) can also be used to determine the qualitative ion concentration distribution in the separator.

However, when graphite is used as the negative electrode, the interlayer distance increases because of lithium intercalation during charging; therefore, a notable volume change occurs compared with that in the hard carbon. In this case, it is difficult to determine the change in the electrolyte concentration change only using $I_{(x, y)}^{\prime} / I_{(x, y)}$ obtained from Eq. (4). Therefore, in this study, the deformation process of the graphite negative electrode that accompanies the charging process was also visualized.

\section{Results}

\subsection{Ion concentration distribution in hard carbon negative electrode}

Figure 5 presents line profiles of $I_{(x)}^{\prime} / I_{(x)}$ in the separator and in the hard carbon negative electrode under constant current charging/discharging conditions of $3 \mathrm{C}$ and $10 \mathrm{C}$ (Figures $5(\mathrm{a})$ and $5(\mathrm{~b})$, respectively). The distribution of $I_{(x)}{ }_{(x)} I_{(x)}$ was determined by averaging the distribution of $I_{(x, y)}^{\prime} I_{(x, y)}$ in the $y$-direction of the X-ray transmission images. The results derived in this manner are consistent with the phenomena normally observed in LIBs, as explained below.

During operation, $I^{\prime}{ }_{(x)} / I_{(x)}$ either increases or decreases upon moving toward the negative-electrode side. For the charging process, an increase of $I_{(x)}^{\prime} / I_{(x)}$ indicates that the ion concentration in the electrolyte decreases. Lithium intercalation occurs in the active material of the negative electrode, and consequently, the concentrations of $\mathrm{Li}^{+}$and $\mathrm{PF}_{6}{ }^{-}$in the electrolyte decrease. The distribution of $I_{(x)}^{\prime} / I_{(x)}$ evidently depends on the rate of charge/discharge. Larger variations emerged at $10 \mathrm{C}$ compared with those at 3C. As the charging/discharging rate increases, the flux of lithium ions through the electrolyte solution increases, and the concentration gradient becomes steep. These results suggest that the change of the ion concentration in a LIB throughout the charging/discharging process can be monitored using the $I_{(x)}^{\prime} / I_{(x)}$ distribution. 
(a) Swelling of graphite electrode

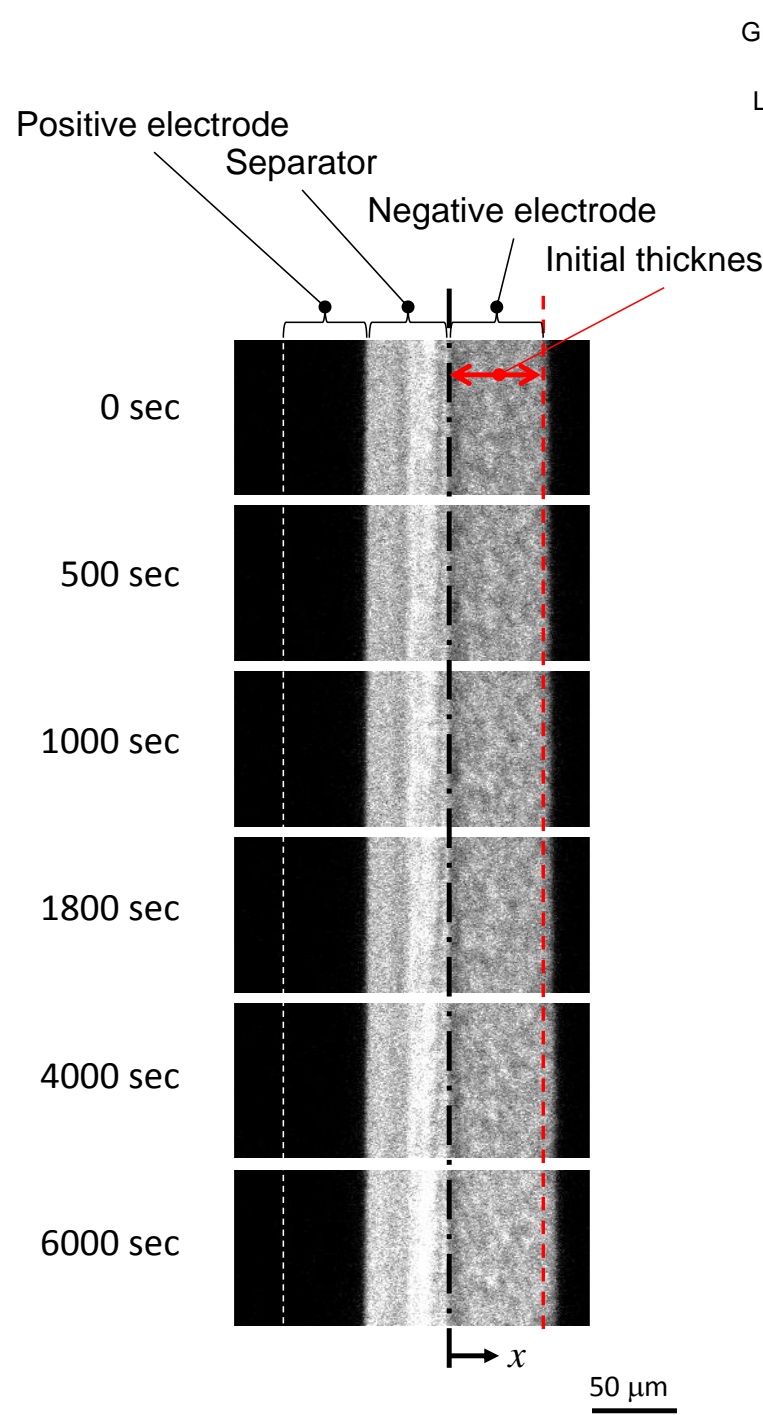

(b) Time variation of graphite electrode thickness

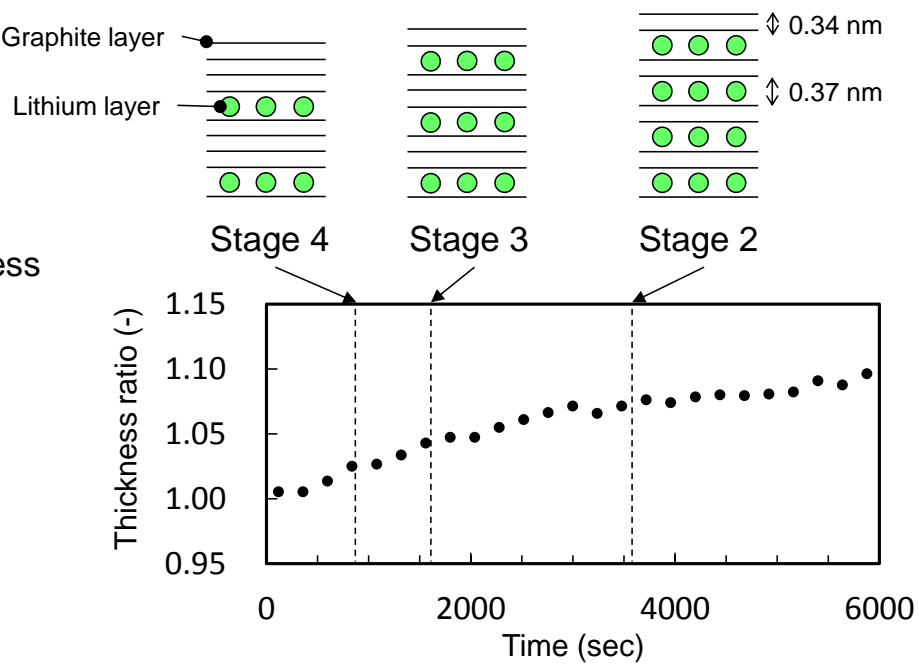

(c) Charging characteristics of LIB

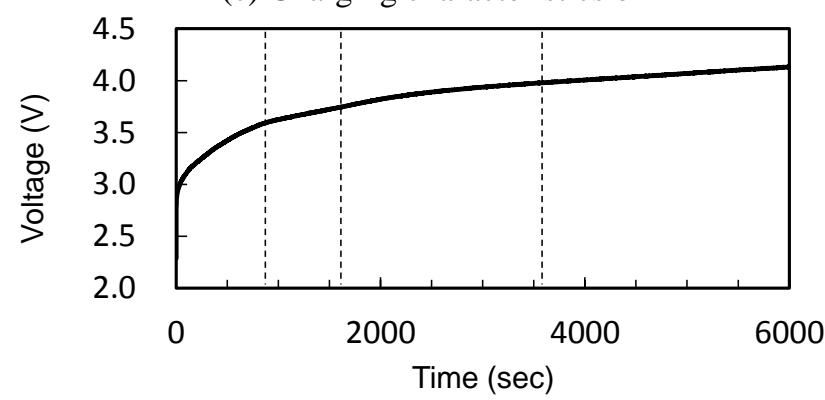

(d) Time derivative of voltage

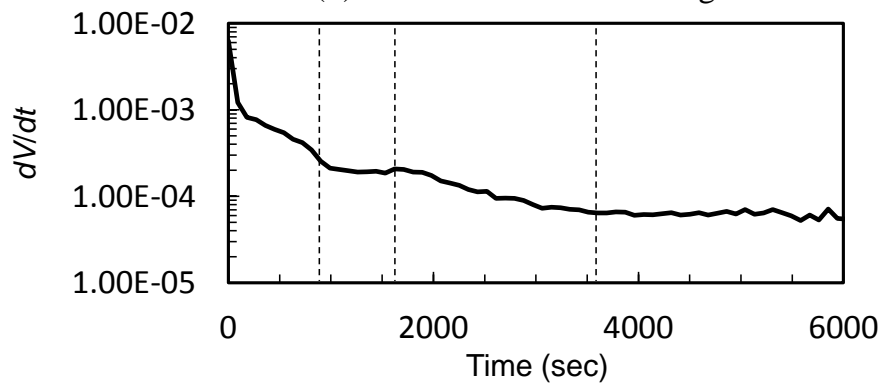

Fig. 6 (a) Swelling of graphite electrode (a partial region in the $y$-direction is shown). (b) Time variation of graphite electrode thickness. (c) Charging characteristics of LIB. (d) Time derivative of voltage.

\subsection{Swelling of graphite negative electrode}

Figure 6(a) shows the time variation of the X-ray transmission images of the graphite negative electrode during the charging process at $0.5 \mathrm{C}$. During the charging process, swelling of the graphite electrode occurred because of lithium intercalation. Lithium intercalation increased the interlayer distance in the graphite negative electrode. As a result, the electrode became thicker and the density of the graphite decreased.

Figure 6(b) shows the time variation of the negative electrode thickness. The thickness ratio is given with respect to the thickness in the initial state. Although the current during the charging process was held constant at $0.5 \mathrm{C}$, the electrode did not linearly expand with time. The increasing trend of the thickness ratio changed at the position indicated by the dashed line in the graph. The position of the dashed line presents the timing of the stage transformation of graphite (Dahn, 1991, Winter et al., 1998, Sethuraman et al., 2010). During the charging process, lithium intercalation does not occur randomly within all the layers of graphite. Lithium is instead regularly inserted at specific intervals, and its arrangement in the graphite varies according to the lithium insertion quantity (charge amount). The stage number is defined according to the insertion state of lithium. Thus, it is considered that the expansion trend changed when stage 
transformation occurs in the graphite.

These experimental results show good agreement with the estimated values of the thickness ratio. The interlayer distance theoretically increases from 0.34 to $0.37 \mathrm{~nm}$ during charging, and the derived thickness ratio is 1.09 (Sato et al., 1995). Correspondingly, the empirical swelling rate between the initial and final states is 1.10.

Stage transformation is also evidenced by the voltage variation during the charging process. The voltage trend is known to change when stage transformation occurs. In Figure 6(c) and (d), the increasing trajectory of the voltage changes slightly at the dashed line, consistent with the time at which the electrode thickness in Figure 6(b) changes expansion trend. These results indicate that low-energy X-ray microscopy can be used to directly measure the change in the electrode thickness resulting from lithium intercalation.

\section{Conclusion}

In this study, in situ visualization of a LIB during charging and discharging was performed using a low-energy $\mathrm{X}$-ray microscopy system. By calculating the ratio of X-ray intensities $\left(I^{\prime}{ }_{(x)} / I_{(x)}\right)$, clear distributions of $\mathrm{Li}^{+}$and $\mathrm{PF}_{6}{ }^{-}$were visualized for a hard carbon negative electrode. Stage transformation caused by lithium intercalation into a graphite negative electrode was also observed. These results suggest that transport phenomena in LIB electrodes can be successfully investigated qualitatively using low-energy X-ray microscopy.

\section{Acknowledgements}

We would like to thank Prof. Shohji Tsushima (Osaka University) for development of the prototype LIB used for visualization in this study.

\section{References}

Bond, T., Zhou, J., Cutler, J., Electrode stack geometry changes during gas evolution in pouch-cell-type lithium ion batteries, Journal of The Electrochemical Society, Vol.164 (2016), pp. A6158-A6162.

Dahn, J. R., Phase diagram of $\mathrm{Li}_{x} \mathrm{C}_{6}$, Physical Review B, Vol.44, No.17 (1991), pp. 9170-9177.

Ebner, M., Marone, F., Stampanoni, M., Wood, V., Visualization and quantification of electrochemical and mechanical degradation in Li ion batteries, Science, Vol.342 (2013), pp.716-719.

Harks, P. P. R. M. L., Mulder, F. M., Notten, P. H. L., Journal of Power Sources, Vol.288, No.92 (2015), pp.92-105.

Sato, A., Takami, N., Ohsaki, T., Electrochemical intercalation of lithium into graphitized carbons, Solid Sate Ionics, Vol.80 (1995), pp. 291-298.

Sethuraman, V.A., Hardwic, L.J., Srinivasan V., Kostecki, R., Surface structural disordering in graphite upon lithium intercalation/deintercalation, Journal of Power Source, Vol.195 (2010), pp. 3655-3660.

Tariq, F., Yufit, V., Kishimoto, M., Shearing, P. R., Menkin, S., Golodnitsky, D., Gelb, J., Peled, E., Brandon, N. P., Three-dimensional high resolution X-ray imaging and quantification of lithium ion battery mesocarbon microbead anodes, Journal of Power Sources, Vol. 248 (2014), pp. 1014-1020.

Tsushima, S., Hung, W., Deevanhxay, P., Kobayashi, G., Kanno, R., Hirai, S., In situ X - ray visualization of the lithiation process in a porous graphite electrode in an operating Li-ion cell, ChemElectroChem, Vol.2 (2015), pp. 1535-1540.

Winter, M., Besenhard, J. O., Spahr, M. E., Novák, P., Insertion electrode materials for rechargeable lithium batteries, Advanced Material, Vol.10 (1998), pp. 725-763. 